\title{
Large gap atmospheric pressure barrier discharges using ferroelectric materials
}

\author{
P. Navascués ${ }^{1}$, A. R. González-Elipe ${ }^{1}$, J. Cotrino ${ }^{1,2}$ and A. Gómez-Ramírez ${ }^{1,2}$ \\ ${ }^{1}$ Laboratory of Nanotechnology on Surfaces. Instituto de Ciencia de Materiales de Sevilla (CSIC- \\ Universidad de Sevilla), Avda. Américo Vespucio 49, 41092 Sevilla, Spain. \\ ${ }^{2}$ Departamento de Física Atómica, Molecular y Nuclear, Universidad de Sevilla, Avda. Reina \\ Mercedes, 41012 Sevilla, Spain.
}

ABSTRACT. This work reports a phenomenological comparative study of atmospheric pressure barrier plasmas using ferroelectric (FBD, ferroelectric barrier discharge) and dielectric (DBD, dielectric barrier discharge) plates to moderate the discharge. Experiments with helium carried out in a parallel plate reactor as a function of applied voltage showed an enhancement of one order of magnitude in the charge transferred through the circuit for FBD operation at large inter-electrode distances. In a similar way to $D B D s$, FBDs rendered a laterally localized arrangement of discrete columnar discharges with a pattern and intensity distribution that depended on operation conditions. Unlike the regular columnar pattern found for DBD operation, discharge columns appear randomly and inhomogenously distributed on the ferroelectric surface for the FBD mode. This particular behavior, the intensity dependence on applied voltage and the peak shape characteristics of the intensity curves have been accounted for by the high capacity of ferroelectric surfaces to randomly accumulate charge and to promote the emission of secondary electrons in the presence of a plasma.

\section{1.- Introduction}

Dielectric barrier discharges (DBD), discovered in the mid of the ninety century by Siemens, ${ }^{1}$ have been employed as ozone generators, ${ }^{2}$ for the removal of volatile organic compounds in $\mathrm{air}^{3,4}$ and, more recently, for the synthesis of chemical compounds (e.g., ammonia, ${ }^{5-7}$ hydrocarbons ${ }^{8}$ and hydrogen ${ }^{9,10}$ ), thin film deposition, ${ }^{11}$ surface treatments ${ }^{12}$ or as plasma actuators for the aerospace industry. ${ }^{13}$ These applications require localized, stable and homogeneous discharge conditions which are not always achieved with atmospheric pressure DBD plasmas. ${ }^{14}$ In fact, although 
homogeneous and uniform DBD discharges can be obtained at low pressures, atmospheric DBDs use to be non-homogeneous and appear as discontinuous columnar patterns or as filamentary discharges. ${ }^{15-18}$

On the other hand, the outstanding properties (e.g., high permittivity, piezoelectricity or pyroelectricity responses or spontaneous reversible polarization) of ferroelectric materials make their use pervasive in capacitors, ${ }^{19}$ transistors, ${ }^{20}$ memory devices, ${ }^{21}$ surface acoustic wave $(\mathrm{SAW})^{22}$ resonators or sensors. ${ }^{23}$ Recently, after measuring a negative capacitance for a PZT ferroelectric thin film, Khan et al. ${ }^{24}$ proposed the incorporation of ferroelectrics to different disruptive plasma technologies. In this line, Johnson et al. ${ }^{25}$ proposed the use of ferroelectric plates in plasma actuators while the incorporation of ferroelectrics in packed-bed plasma reactors proved to increase their chemical efficiency for the synthesis of ammonia, conversion of $\mathrm{CO}_{2}$ or the reforming of hydrocarbons. ${ }^{6,9,26}$ The higher dielectric constant of ferroelectrics with regard to classical dielectrics (e.g., quartz or alumina) has been claimed to contribute to the enhancement of process yield. ${ }^{4,27}$ However, beyond this correlation, more systematic analysis are still needed to determine the actual behavior of FDB plasmas and the magnitude of plasma intensity (i.e, the transferred charge) when using ferroelectrics in different reactor configurations. The analysis of the discharge behavior at low frequencies, low voltages and large inter-electrode distances is crucial for the industrial use of DBD/FBD discharges, and especially for those applications having gained attention recently as seed treatment or plasma medicine. ${ }^{28,29}$ The present work addresses a series experimental issues whose determination is deemed critical for a better understanding of FBD plasmas: i) I-V dependence, ii) characteristics (filamentary or glow ${ }^{15}$ ) of the obtained FBD plasmas and iii) heterogeneous distribution of plasma zones on operation conditions and type of barrier material. ${ }^{15,30-32}$ In particular, we compare the electrical behavior and the spatial distribution of plasma in a parallel plate reactor moderated with ferroelectric and dielectric plates. Parameters such as inter-electrode distance and applied voltage, known to control the charge accumulation on ferroelectric surfaces ${ }^{33,34}$ and their capacity to emit electrons, ${ }^{35}$ have been systematically varied as running parameters in this study. 


\section{2.-Experimental section}

Experiments have been carried out in a plasma reactor consisting of a chamber with two parallel stainless steel plate electrodes on which either ferroelectric ( $\mathrm{LiNbO}_{3}$, both size polished, $0.5 \mathrm{~mm}$-width and cut at 128 o with respect to the $\mathrm{Y}$-axis, Roditi International Corporation, London) or dielectric (fused silica from Sceram, France) plates were fixed. The relative dielectric constant of fused silica is similar to that of quartz plates (i.e., 4.60 and 4.51 , along parallel or perpendicular directions to the optical axis $^{36}$ ) and much lower than that of $\mathrm{LiNbO}_{3}$ (main axis equivalent values are $\mathrm{T}_{11}=85.2, \mathrm{~T}_{22}=85.2$ and $\mathrm{T}_{33}=28.7$, as provided by the supplier). The upper electrode was high voltage activated while the bottom electrode was grounded. Time evolution of the charge transferred during the discharge, $Q_{t}(t)$, and consumed power, $P(w)$, were determined by time integration of the measured $I(t)$ and from the area of the Lissajous $(Q(t)-V(t))$ curve, respectively (see supplementary material S1). Trying to optimize the experimental conditions and to reduce the consumed power, both the input signal frequency $(1 \mathrm{kHz})$ and the applied voltage (varying from 0.5 to $3 \mathrm{kV}$ ) were much smaller than typical frequencies and voltages used in DBD. ${ }^{35}$ The discharge current was measured by a coil (Pearson current monitor, Model 2877) in series with the reactor and at ground side of the equivalent circuit. $V(t)$ was measured using a high voltage probe directly connected to the active electrode. Both $I(t)$ and $V(t)$ signals were collected with an oscilloscope (Tektronix TDS2001C). A more detailed description of the electrical layout is supplied as supplementary material S2.

The ferroelectric plate (100 mm wafer diameter) was larger than the metal electrode ( $80 \mathrm{~mm}$ diameter) to avoid the formation of micro-discharges at the electrodes edges. The inter-electrode distance was varied with a micrometric screw connected to the top electrode. All the experiments were carried out at room temperature and atmospheric pressure. Helium, selected as plasma gas because its well-recognized capacity to form glow DBD plasmas, ${ }^{33,37}$ was delivered to the chamber through apertures situated over the active electrode, and left the reactor through a lateral port. It is noteworthy that, due to the low chamber vacuum reached before experimental measurements $\left(10^{-2}\right.$ mbar), the presence of some nitrogen impurities that can contribute to obtain a more homogenous discharge as a consequence of Penning ionization processes ${ }^{38}$ cannot be 
neglected. Gas flow rate was maintained constant at $2.0 \mathrm{~L} / \mathrm{min}$. Time-integrated pictures of the plasma were taken with a Canon EOS 5D-III camera (exposure time $1 \mathrm{~ms})$.

\section{3.-Results and Discussion}

\section{1.-Characterization of expanded plasma discharges}

Figure 1 (a) shows the characteristic $l(t)-V(t)$ curves and the temporal evolution of transferred charge when using ferroelectric plates (i.e., in FBD mode) for a $9.8 \mathrm{~mm}$ inter-electrode spacing and a $1.98 \mathrm{kV}$ amplitude sinusoidal voltage. This value was the minimum required to switch-on a plasma completely filling the electrodes region. For a given gap, we denote this switch-on value as threshold voltage and the obtained plasma as expanded discharge extending over the whole electrode surface (note that this expanded plasma is usually columnar and that, once formed, the discharge can be maintained at lower voltages depicting a different pattern of plasma columns, see section 3.2).

Figure 1 (b) shows a similar plot of $V(t)$ and $I(t)$ curves for the reactor operated with a quartz plate (i.e., in DBD mode). The discharge electrical behavior was qualitatively equivalent to that of FBD, with the significant difference of a smaller width and much lower intensity of the $\mathrm{I}(\mathrm{t})$ peak. In the two cases, it is noteworthy that the smooth shape and discrete time spam of the intensity curve for $\mathrm{l}(\mathrm{t}) \neq 0$ discard the formation of micro-discharges during either FBD or DBD operation and suggest that plasma discharge behaves like an APGD (atmospheric pressure glow discharge). ${ }^{33,35,39}$ Nevertheless, the discharge appearance was different when using dielectric materials, whereby the plasma column distribution was more ordered and homogeneous, as described in section 3.2.

The shape and other features of the $I(t)-V(t)$ curves represented in Figure 1 may be used to identify characteristic discharge events that can be described in the following terms: 
(a) At $V(t)=0$ (point "1") no current is flowing through the circuit, although the ferroelectric plate may keep some charge from a previous excitation cycle.

(b) Upon plasma ignition, current sharply increases while, simultaneously, at breakdown point " 2 ", $\mathrm{V}(\mathrm{t})$ sharply drops to immediately recover its sinusoidal shape (see the inset in Figure 1(a)). Similar distortions have been observed in other FBD experiments ${ }^{25}$ and attributed to a rapid realignment of accumulated charge during ferroelectric switching. We assume that a modification in the space charge distribution may create a strong electric field at the electrode surface and provoke the observed sudden voltage drop. Similar, though less abrupt, distortions were found in the DBD experiments of Figure 1 (b). The equivalent $\mathrm{FBD}$ and $\mathrm{DBD}$ behaviors suggest that, rather than resulting from ferroelectric switching, the observed voltage distortions under plasma ignition must be due to differences in the charge re-distribution on the barrier materials.

(c) After ignition, current continuously increases up to reach its maximum value at point " 3 " (74 mA in Figure 1 (a) and 18 mA in Figure 1 (b)). After reaching this point, $l(t)$ progressively decreases until plasma vanishes at point " 4 ". Then, the transferred charge $\left(Q_{t}\right)$ remains constant till the polarity changes and the plasma ignites again (point " 5 "). The discharge behavior is mirror-symmetrical with respect to the first half period, making that $Q_{t}(t)$ becomes zero again due to the opposite sign of $I(t)$. As shown by Pipa et al., ${ }^{40}$ the transferred charge must be equivalent to the accumulated charge on the barrier surfaces.

(d) The process repeats continuously following the periodicity of the excitation signal, $V(t)$. 

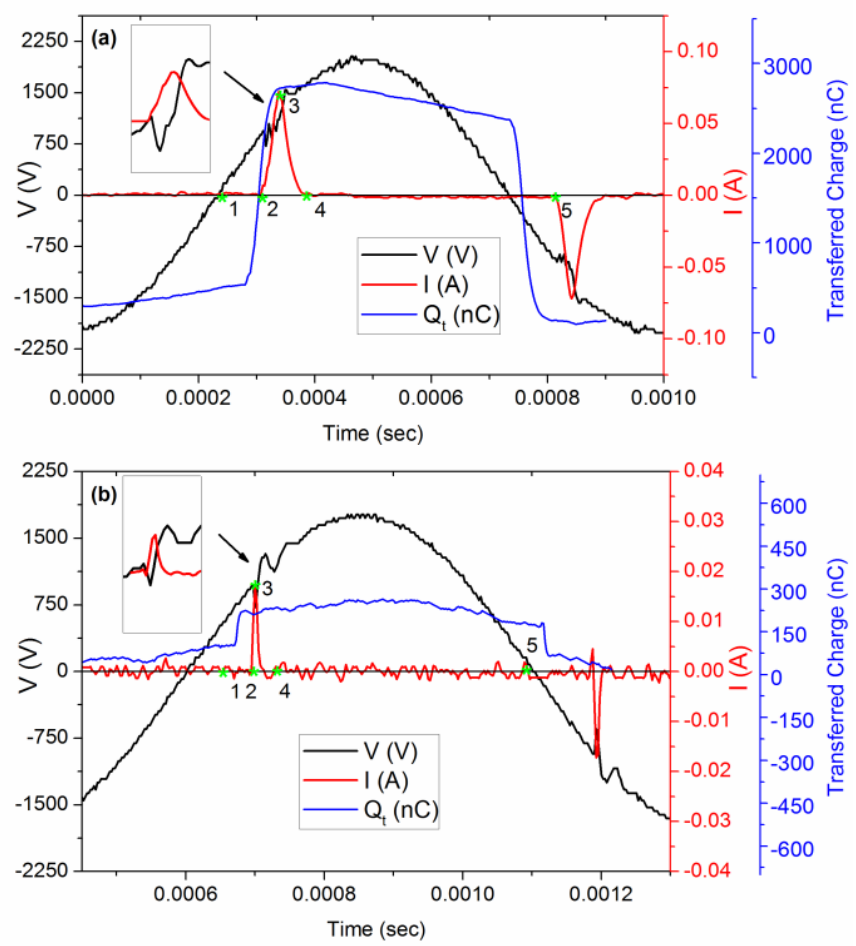

Figure 1. (a) I(t)/V(t)/Q $Q_{t}(t)$ curves for an inter-electrode distance $9.8 \mathrm{~mm}$ and $\mathrm{LiNbO}_{3}$ as barrier material. (b) Idem for quartz as barrier material. The insets show a timeexpanded view of the voltage drop and intensity evolution upon plasma ignition.

To highlight the different capacity of charge accumulation and discharge behavior of FBD and DBD modes, we represent in Figure 2 the Lissajous curves deduced from the previous $I(t)-V(t)$ plots. The comparison of these two curves clearly sustains that in FBD mode plasma transfers much more charge than in DBD mode. The much lower magnitude of the transferred charge in DBD agrees with the smaller dielectric constant of fused silica and its lower capacity to accumulate charge (e.g., $2470 \mathrm{nQ}$ on $\mathrm{LiNbO}_{3}$ against $170 \mathrm{nQ}$ on fused silica as determined from the Lissajous curves in Figure 2). 


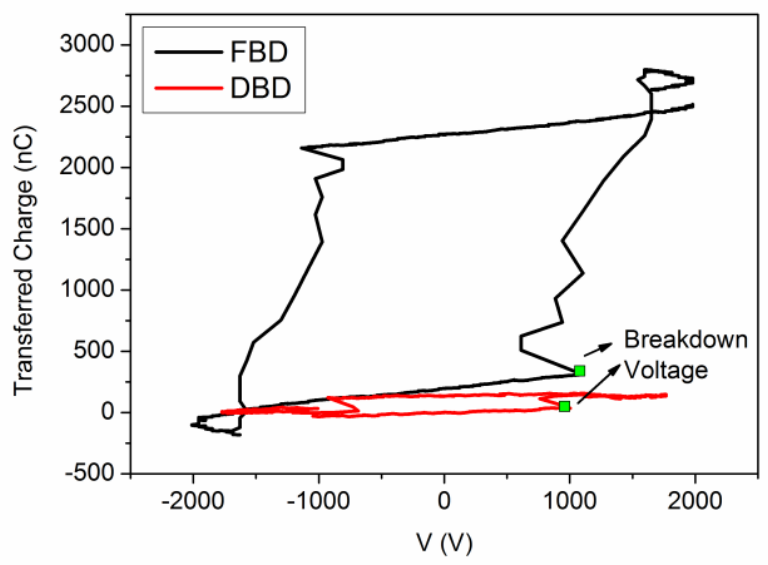

Figure 2. Lissajous plots calculated from the $\mathrm{I}(\mathrm{t})-\mathrm{V}(\mathrm{t})$ plots in Figure 1.

It is also noteworthy in these plots that, according to the analysis above of $I(t)-V(t)$ curves (i.e. bullet point b), the breakdown voltage (i.e., the voltage required to induce current flow between electrodes, whatever its spatial distribution, see below) corresponds the squared green dots in the Lissajous curves represented in Figure 2. This assignment slightly differs from the common attribution of breakdown voltage to the point where the Lissajous curve cuts the X-axis. ${ }^{40}$ The difference between these two assignments would be small for square shaped curves and high capacitive materials, but might be quite significant in processes where Lissajous curves are elliptical. ${ }^{9}$ In this line, it is also remarkable that the sharp voltage drop observed at breakdown (c.f., inset in Figure 1a) renders a temporary negative capacitance $(d Q / d V<0)$, as deduced from the negative slope of the Lissajous curve at this point (Figure 2). ${ }^{24}$

Additional experimental evidences of a higher efficiency of FBD vs. DBD are reported in Figure 3. For both FBD and DBD plasmas, this figure displays the variation of the applied threshold voltage and the maximum value of transferred charge during one electrical cycle as a function of inter-electrode distance. As observed, the threshold voltage is approximately the same in both cases, while transferred charge values are higher for the FBD, independently of the electrode separation. It is apparent that the slope of the FBD transferred charge curve experiences a slight increase for interelectrode distances higher than $7 \mathrm{~mm}$. We attribute this increase to a significant 
contribution from particle induced emission of secondary electrons (i.e., electrons emitted when energetic particles reach the ferroelectric/dielectric plates), in addition to electrons coming from gas ionization processes. This phenomenon would be much larger for FBDs, where the direct electron emission from the ferroelectric, related with its secondary emission coefficient $(\gamma)$, should be much higher than that due to ionization of the plasma gas. Indeed, for both kinds of materials, the current curve shape in Figure 1, coming down to cero just after reaching the maximum, point to a discharge controlled by secondary emission processes instead of ionization processes, likely characterized by a longer time discharge. In agreement with Massines et al., ${ }^{35}$ secondary electrons modulated by plasma would deploy a positive charge space along the inter-electrode gap and contribute to the observed voltage drop at breakdown when current flows between the two electrode plates (see insets in Figure 1). Plasma induced electron emission have been observed for other barrier materials ${ }^{41}$ (ferroelectric or not). In ferroelectrics, mechanisms of electron emission due to domain switching ${ }^{42}$ and the presence of high local electric fields on the ferroelectric surface ${ }^{43}$ may also contribute to $I(t)$. Recently, Johnson et al. have determined that secondary electron emission from FBD surfaces may reach values up to $80 \mathrm{~mA},{ }^{25}$ lower than some of the current flows found in the present work (see supplementary material, Figure S3). Due to the complexity of the phenomena taking place at ferroelectric charged surfaces, a comprehensive explanation of plasma induced secondary electron emission from ferroelectrics is still missing, including the possible contribution of other emission phenomena besides secondary electron emission. In the case of dielectric materials several authors have analyzed these issues, ${ }^{35}$ pointing to the formation of a positive column and long-living species as key factors to enhance secondary emission. 


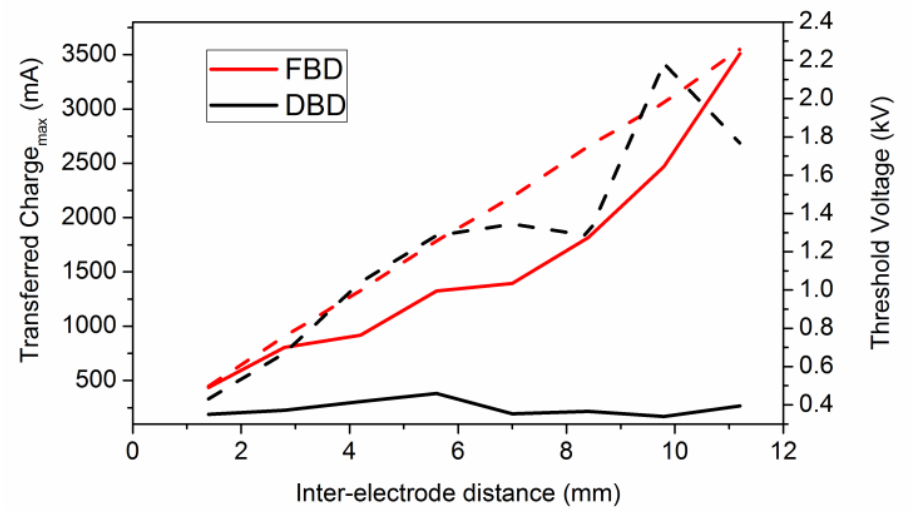

Figure 3. Evolution of transferred charge (continuous line) and threshold voltage (dash line) with the inter-electrode distance for both FDB (red lines) and DBD (black lines).

The active role of the ferroelectric surface in contributing to plasma formation is further demonstrated by analyzing the time evolution of the current peak in successive ignition events. Figure 4 (a) shows a typical behavior of the $I(t)$ curve for successive switching cycles of the plasma discharge for an inter-electrode distance of $9.8 \mathrm{~mm}$. It appears that, while the $V(t)$ curve shape is stable, the height of the current peak continuously decreases during the reported five positive and negative ignition events (after the third/fifth period, depending on conditions, the height of the intensity curve recovers always its maximum to reproduce the process in a cyclic way). Yet, a closer inspection to the peaks shapes reveals that the integrated charge does not change from the first to the fifth positive event, but there is an increase in peak width and the appearance of a small repetition feature separated by $0.1 \mathrm{~ms}$ from the main peak (Figure 4 (b)). This evolution reveals a kind of memory effect which we do not observed in DBD mode under our experimental conditions, probably because of the high surface charge trapping capacity in FBD and the related increase in the secondary electron probability. ${ }^{35}$ 

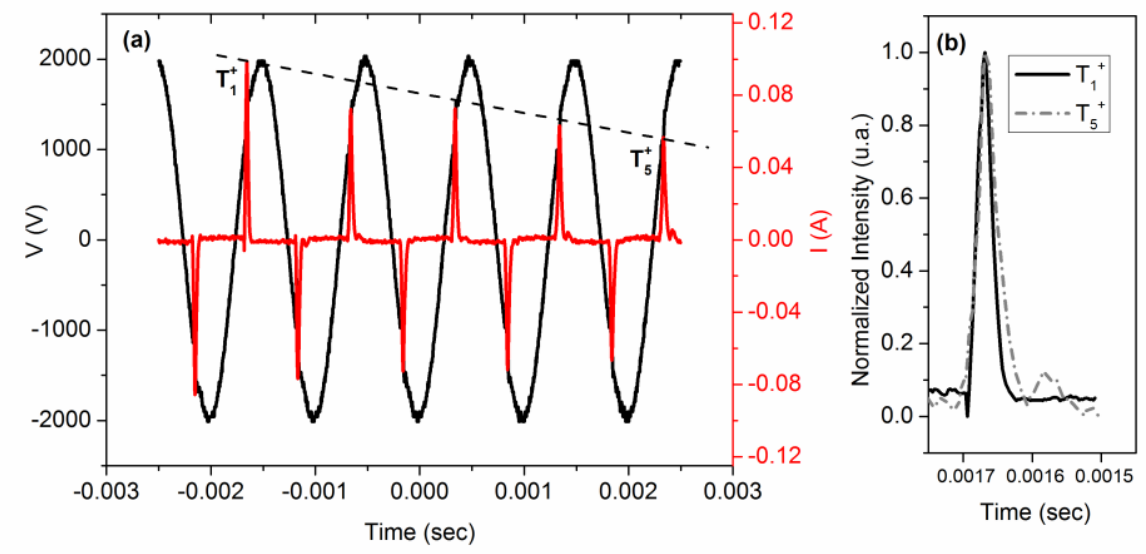

Figure 4. (a) Plot of the $V(t)$ and $I(t)$ curves during five alternant periods during a FBD experiment for an inter-electrode distance of $9.8 \mathrm{~mm}$. (b) Normalized plot of the $\mathrm{T}_{1}{ }^{+}$ and $\mathrm{T}_{5}{ }^{+}$peaks to highlight their different width and shape.

\section{2.-Lateral distribution of plasma}

The formation of columnar patterns in atmospheric pressure DBD plasmas is a wellknown phenomenon ${ }^{30-32,44}$ that has been associated with local variations of capacitance and potential along the discharge zone, and with the loss of discharge homogeneity due to the interaction of charged particles with the dielectric surfaces. ${ }^{45,46} \mathrm{Xu}$. et al. ${ }^{47}$ have numerically analyzed these changes, accounting for the plasma patterns formed at low pressure DBD discharges. In agreement with these works, a patterned columnar distribution of plasma was also found during DBD and FBD operation of our reactor (note however the quite different intensity of the discharges as illustrated in Figure 1). Figure 5 shows expanded discharge photographs for the two analyzed cases at the threshold voltage and an inter-electrode separation of $9.8 \mathrm{~mm}$. Although columnar-like structures are formed both for FBD and DBD, the number of columns is higher in the former case where it also depicts random spatial distribution. For DBDs, plasma columns appear as an organized and symmetrical distribution where columns are equidistance. The more random distribution in FBD could be correlated with variations in surface charge distribution on the ferroelectric material where a higher capacity to accumulate charge could give rise to inhomogeneities in its surface 
distribution due to free charge mobility and hence to the appearance of new columns arbitrary positioned onto the surface.

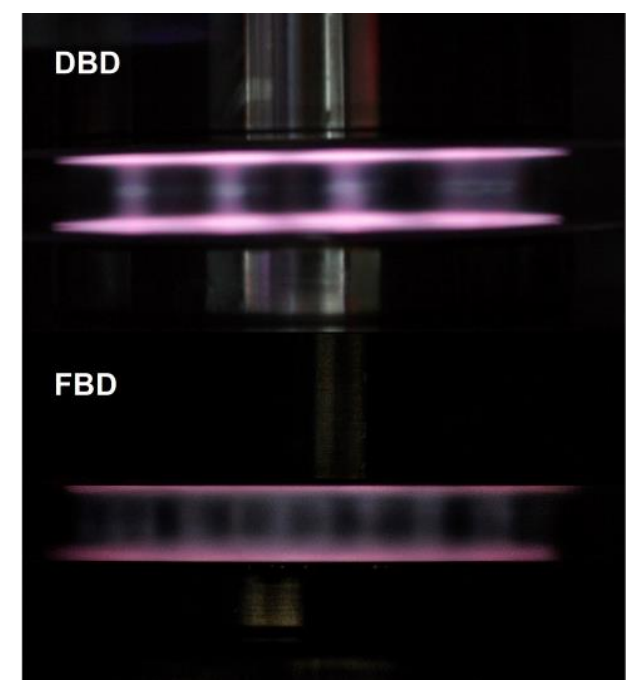

Figure 5. Columnar plasma distribution for DBD (top) and FBD (bottom) discharges (interelectrode distance $9.8 \mathrm{~mm}, V_{\text {threshold }} 1.77 \mathrm{kV}$ and $1.98 \mathrm{kV}$ for DBD and FBD respectively) showing a more heterogeneous pattern in the latter case.

In addition to the previous analysis of FBD vs. DBD for expanded plasma conditions, i.e., when the plasma has been ignited at the threshold voltage, we have also investigated the columnar lateral distribution of plasma in FBD when lowering the applied voltages from this state.

Figure 6 reports the FBD intensity curves and plasma characteristics for several voltages at a fixed gap of $9.8 \mathrm{~mm}$. According to this figure, at the threshold voltage of $1.98 \mathrm{kV}$, plasma consists of a distribution of multiple plasma columns inhomogeneously spread onto the electrode surface. Then, when lowering the voltage, plasma distribution transforms into others with only a few isolated plasma columns (e.g., three columns appeared at $1.84 \mathrm{kV}$ and only one at $1.72 \mathrm{kV}$ ). A closer look to the image of these individual columns reveals that, independently on its number, their vertical profile has consecutive dark and bright regions mimicking the behavior of a conventional glow discharge. ${ }^{37}$ A glow character of these plasma columns, independently on their lateral distribution, is further supported by the similar shapes of $I(t)$ curves at decreasing voltages (c.f. Figure 6). It is also noteworthy that in our experiment the already referred progressive decrease in the number of plasma 
columns with the applied voltage could be only observed for FBD, but not for DBD plasmas. In agreement with $\mathrm{Hao}$ et $a l .{ }^{48}$, in the DBD experiment, the transition to isolated well-separated plasma columns could only be observed by slowly decreasing the voltage. Again, this difference must be associated with the higher capacity of charge accumulation on the surface of the ferroelectric plate electrodes.

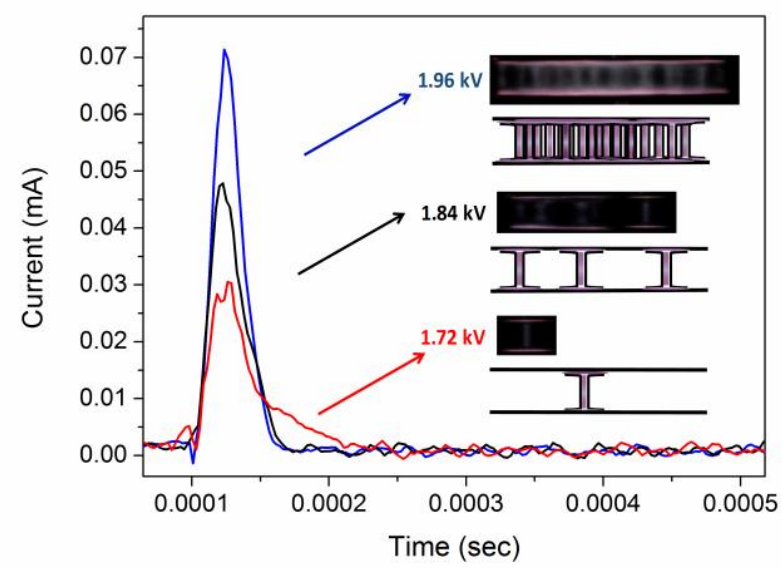

Figure 6. Evolution of $I(t)$ curves recorded during a half cycle for progressively smaller applied voltages and a gap of $9.8 \mathrm{~mm}$ in FBDs. The corresponding photographs and plasma schemes at the right hand side of the figure illustrate the evolution of plasma distribution up to its extinction.

The complex evolution of columnar pattern in FBD was rather complex and highly dependent on working conditions. The series of photographs in Figure 7 (a), taken at the threshold voltages required to ignite an expanded discharge, show that plasma distribution varied with electrode separation and its associated threshold voltage. For small inter-electrode separations (1.4-2.8 $\mathrm{mm})$, plasma spread homogeneously through the whole gap with no clear hints of columns formation. Then, for larger gaps, it broke up into separated and less numerous columns up to an inter-electrode distance of $7.0 \mathrm{~cm}$ or larger, a value for which the number of columns remained virtually constant. To explain the decreasing number of discharge channels as gap distance increases it is necessary to take into account the evolution of the accumulated charge and its spatial distribution. The longer the gap the higher the accumulated charge needed to ignite the discharge, in fact, transferred charge 
increases as the distance does (see Figure 3). Charges would tend to be accumulated at some points on the barrier material surfaces in order to reach the necessary conditions to ignite the discharge, giving rise to several discharge channels. As the gap distance increases the number of charges accumulated in each point needs to be higher to initiate ignition, resulting in a reduced number of current channels. In parallel to these changes in the lateral distribution of plasma, the number of well-defined intensity $I(t)$ peaks during a half voltage period varied from three for the smallest inter-electrode gaps to two and one at larger gaps (c.f., Figure $7(b))$. This finding and the different time evolution of $Q_{t}$ clearly demonstrate that various breakdown events can be triggered during a half period, particularly when there is a small separation between electrodes. This is a consequence of the glow character of the discharge, the fast decay of the current peak and the low frequency used for these experiments. This discharge behavior, together with the memory effect accounted before, are clear demonstrations of interplay between charge accumulation and plasma ignition phenomena when using ferroelectric materials as electrodes. It is also noteworthy that the low power required to ignite the FBDs (varying from $0.20 \mathrm{~W}$ to $7.48 \mathrm{~W}$ for 1.4 $\mathrm{mm}$ and $11.2 \mathrm{~mm}$ gaps, respectively, see supplementary material $\mathrm{S} 4)$ and the fulfilment of the Meek criterion ${ }^{33}$ for all investigated discharges support the glow character of these plasmas, ${ }^{35}$ independently of the number of events per period. We must stress that a columnar distribution of plasma is compatible with a glow ionization $^{33}$ and simply reveals that charge density accumulation on ferroelectric surface and gas volume vary laterally with operating conditions. Thus, since the shape of the intensity/transferred charge curves was similar in the two cases, most important difference between FBD and DBD is the much smaller intensity of the transferred charge and the more ordered plasma columnar distribution in the latter. Such a difference must be explained in terms of charge surface distribution, which is higher and more inhomogeneous onto the ferroelectric material. 
(a)

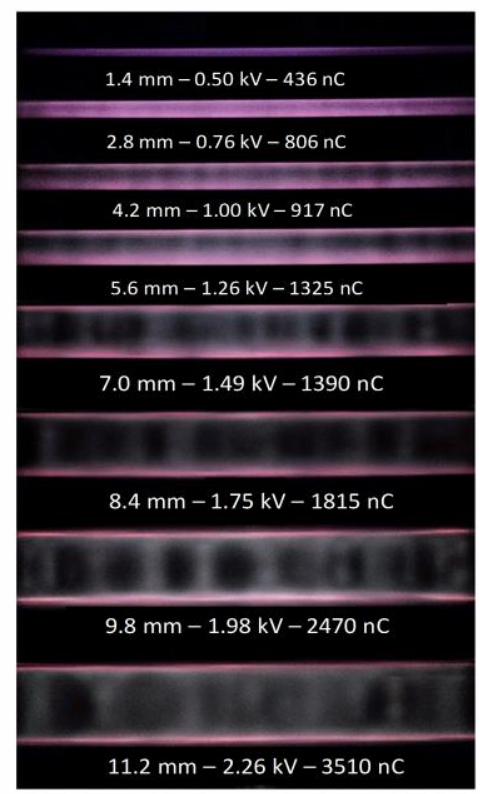

(b)

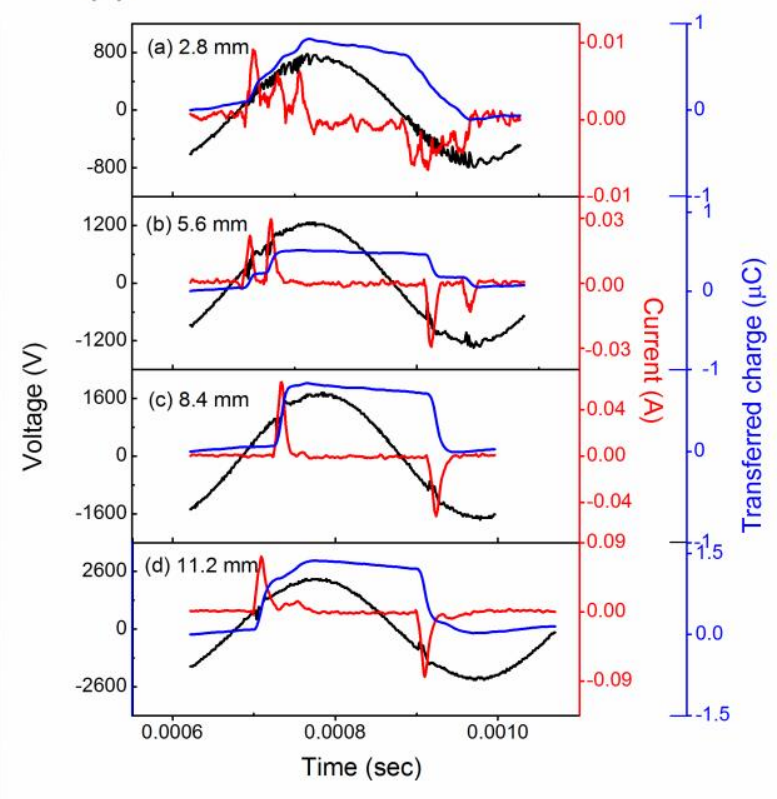

Figure 7. (a) Photographs of a FBD taken for different inter-electrode distances and threshold voltages. (b) Selected $I(t), V(t)$ and $Q_{t}(t)$ curves determined for these discharges.

\section{4.-Conclusions}

The phenomenological description of plasma discharges in a parallel plate FBD reactor has shown that charge injection into the plasma determines both current intensity and plasma spatial distribution. DBD and FBD present similar threshold voltages but the higher polarizability of ferroelectric materials determines a much higher charge accumulation onto the electrode surface and hence, a higher discharge current. In addition, the higher capacity for charge accumulation on the ferroelectric surface is deemed to be the main factor responsible for a non-equidistant plasma column distribution. The underlying factors leading to the found increase of current density must be related with basic physical processes such as the emission of secondary electrons and their injection into the plasma. We believe that the intensity enhancement found in FBD reactors, the low consumed power to sustain the discharges and the possibility to tune their electrical behavior at quite high interelectrode separations are key features to develop new atmospheric pressure plasma devices for advanced applications as seed treatment and plasma medicine. 


\section{SUPPLEMENTARY MATERIAL}

See supplementary material S1 for transferred charge calculation, S2 for a detailed description of the reactor, S3 for a study of the maximum current vs inter-electrode distance in both cases (FBD and DBD) and S4 for the FBD Lissajous plots at different inter-electrode distances.

\section{ACKNOWLEDGMENTS}

We thank EU-FEDER funds and the MINECO (projects MAT2013-40852-R, MAT201679866-R, MINECO-CSIC 201560E055) and Junta de Andalucía (project P12-2265 MO) for financial support.

\section{REFERENCES}

1. W., S. Über die elektrostatische Induction und die Verzögerung des Stroms in Flaschendrähten. Ann. Phys. 178, 66-122 (2006).

2. Chen, H. L., Lee, H. M., Chen, S. H. \& Chang, M. B. Review of Packed-Bed Plasma Reactor for Ozone Generation and Air Pollution Control. Ind. Eng. Chem. Res. 47, 2122-2130 (2008).

3. Hyun- Ha, K. Nonthermal Plasma Processing for Air- Pollution Control: A Historical Review, Current Issues, and Future Prospects. Plasma Process. Polym. 1, 91-110 (2004).

4. Gómez-Ramírez, A., M. Montoro-Damas, A., Rodríguez, M. A., R. GonzálezElipe, A. \& Cotrino, J. Improving the pollutant removal efficiency of packed-bed plasma reactors incorporating ferroelectric components. Chem. Eng. J. 314, 311319 (2017).

5. Gómez- Ramírez, A., Montoro- Damas, A. M., Cotrino, J., Lambert, R. M. \& González- Elipe, A. R. About the enhancement of chemical yield during the atmospheric plasma synthesis of ammonia in a ferroelectric packed bed reactor. Plasma Process. Polym. 14, 1600081 (2016).

6. Gómez-Ramírez, A; Cotrino, J; Lambert, R. M. and González-Elipe, A. R. Efficient synthesis of ammonia from $\mathrm{N} 2$ and $\mathrm{H} 2$ alone in a ferroelectric packedbed DBD reactor. Plasma Sources Sci. Technol. 24, 65011 (2015).

7. Peng, P. et al. A review on the non-thermal plasma-assisted ammonia synthesis technologies. J. Clean. Prod. 177, 597-609 (2018).

8. Gómez-Ramírez, A., Rico, V. J., Cotrino, J., González-Elipe, A. R. \& Lambert, 
R. M. Low Temperature Production of Formaldehyde from Carbon Dioxide and Ethane by Plasma-Assisted Catalysis in a Ferroelectrically Moderated Dielectric Barrier Discharge Reactor. ACS Catal. 4, 402-408 (2014).

9. Montoro-Damas, A. M., Brey, J. J., Rodríguez, M. A., González-Elipe, A. R. \& Cotrino, J. Plasma reforming of methane in a tunable ferroelectric packed-bed dielectric barrier discharge reactor. J. Power Sources 296, 268-275 (2015).

10. Chen, H. L., Lee, H. M., Chen, S. H., Chao, Y. \& Chang, M. B. Review of plasma catalysis on hydrocarbon reforming for hydrogen productionInteraction, integration, and prospects. Appl. Catal. B Environ. 85, 1-9 (2008).

11. Massines, F., Gherardi, N., Fornelli, A. \& Martin, S. Atmospheric pressure plasma deposition of thin films by Townsend dielectric barrier discharge. Surf. Coatings Technol. 200, 1855-1861 (2005).

12. Wagner, H.-E. et al. The barrier discharge: basic properties and applications to surface treatment. Vacuum 71, 417-436 (2003).

13. Erfani, R., Zare-Behtash, H., Hale, C. \& Kontis, K. Development of DBD plasma actuators: The double encapsulated electrode. Acta Astronaut. 109, 132-143 (2015).

14. Fang, Z., Qiu, Y., Zhang, C. \& Kuffel, E. Factors influencing the existence of the homogeneous dielectric barrier discharge in air at atmospheric pressure. J. Phys. D. Appl. Phys. 40, 1401 (2007).

15. Kogelschatz, U. Filamentary, patterned, and diffuse barrier discharges. IEEE Trans. PLASMA Sci. 30, 1400-1408 (2002).

16. Naudé, N., Cambronne, J.-P., Gherardi, N. \& Massines, F. Electrical model and analysis of the transition from an atmospheric pressure Townsend discharge to a filamentary discharge. J. Phys. D. Appl. Phys. 38, 530 (2005).

17. Dong College of Physics Science and Technology, Hebei University, Baoding 071002 (China)], L. [Hebei K. L. of O.-E. I. and M. Simulation of stationary glow patterns in dielectric barrier discharges at atmospheric pressure. (2014). doi:10.1063/1.4903460

18. Hao, Y., Zheng, B. \& Liu, Y. Columnar discharge mode between parallel dielectric barrier electrodes in atmospheric pressure helium. Phys. Plasmas 21, 13503 (2014).

19. Gao, W. et al. Room-Temperature Negative Capacitance in a FerroelectricDielectric Superlattice Heterostructure. Nano Lett. 14, 5814-5819 (2014).

20. Appleby, D. J. R. et al. Experimental Observation of Negative Capacitance in Ferroelectrics at Room Temperature. Nano Lett. 14, 3864-3868 (2014). 
21. Kundu, S. et al. Integration of lead-free ferroelectric on HfO2/Si (100) for high performance non-volatile memory applications. Sci. Rep. 5, 8494 (2015).

22. Izyumskaya, N. et al. Processing, Structure, Properties, and Applications of PZT Thin Films. Crit. Rev. Solid State Mater. Sci. 32, 111-202 (2007).

23. Muralt, P. Ferroelectric thin films for micro-sensors and actuators: a review. $J$. Micromechanics Microengineering 10, 136-146 (2000).

24. Khan, A. I. et al. Negative capacitance in a ferroelectric capacitor. Nat. Mater. 14, 182 (2014).

25. Johnson, M. J. \& Go, D. B. Ferroelectric crystals for the low-voltage operation of surface dielectric barrier discharges. Appl. Phys. Lett. 105, 264102 (2014).

26. Xu, S., Whitehead, J. C. \& Martin, P. A. CO2 conversion in a non-thermal, barium titanate packed bed plasma reactor: The effect of dilution by Ar and N2. Chem. Eng. J. 327, 764-773 (2017).

27. Li, H. \& Subramanyam, G. Capacitance of thin-film ferroelectrics under different drive signals. IEEE Trans. Ultrason. Ferroelectr. Freq. Control 56, 1861-1867 (2009).

28. Gómez-Ramírez, A. et al. Surface chemistry and germination improvement of Quinoa seeds subjected to plasma activation. Sci. Rep. 7, 5924 (2017).

29. Fridman, A. \& Friedman, G. Plasma Medicine. (Wiley, 2012).

30. Kogelschatz, U. Collective phenomena in volume and surface barrier discharges. J. Phys. Conf. Ser. 257, 12015 (2010).

31. Boeuf, J. P., Bernecker, B., Callegari, T., Blanco, S. \& Fournier, R. Generation, annihilation, dynamics and self-organized patterns of filaments in dielectric barrier discharge plasmas. Appl. Phys. Lett. 100, 244108 (2012).

32. Boeuf, T. C. and B. B. and J. P. Pattern formation and dynamics of plasma filaments in dielectric barrier discharges. Plasma Sources Sci. Technol. 23, 54003 (2014).

33. Sanden, S. A. S. and P. A. P. and M. C. and E. M. van V. and H. de V. and R. M. J. P. and M. C. M. van de. On the formation mechanisms of the diffuse atmospheric pressure dielectric barrier discharge in CVD processes of thin silicalike films. Plasma Sources Sci. Technol. 18, 45021 (2009).

34. Bogaczyk, M., Wild, R., Stollenwerk, L. \& Wagner, H.-E. Surface charge accumulation and discharge development in diffuse and filamentary barrier discharges operating in He, N 2 and mixtures. J. Phys. D. Appl. Phys. 45, 465202 (2012).

35. Massines, F., Gherardi, N., Naudé, N. \& Ségur, P. Recent advances in the 
understanding of homogeneous dielectric barrier discharges. Eur. Phys. J. Appl. Phys. 47, (2009).

36. Bottom, V. E. Dielectric Constants of Quartz. J. Appl. Phys. 43, 1493-1495 (1972).

37. Massines, F. et al. Experimental and theoretical study of a glow discharge at atmospheric pressure controlled by dielectric barrier. J. Appl. Phys. 83, 2950 2957 (1998).

38. Influence of impurities on the uniform atmospheric-pressure discharge in helium. Phys. Plasmas 12, 23503 (2005).

39. Massines, F. \& Gouda, G. A comparison of polypropylene-surface treatment by filamentary, homogeneous and glow discharges in helium at atmospheric pressure. J. Phys. D. Appl. Phys. 31, 3411 (1998).

40. Pipa, A. V, Koskulics, J., Brandenburg, R. \& Hoder, T. The simplest equivalent circuit of a pulsed dielectric barrier discharge and the determination of the gas gap charge transfer. Rev. Sci. Instrum. 83, 115112 (2012).

41. Fridman, A. Plasma Chemistry. (Cambridge University Press, 2008). doi:DOI: 10.1017/CBO9780511546075

42. Sidorkin, A. S. \& Darinskii, B. M. Electron emission from ferroelectric plate stimulated by switching. Appl. Surf. Sci. 111, 325-328 (1997).

43. Biedrzycki, H. W. J. and J. H. and K. Dielectric barrier discharge at the triglycine sulfate crystal surface: the role of the electric field of the domain structure. $J$. Phys. D. Appl. Phys. 47, 305202 (2014).

44. Fan, W. et al. Formation of side discharges in dielectric barrier discharge. Sci. Rep. 7, 8368 (2017).

45. Behnke, Y. B. G. and V. A. M. and J. B. and J. F. Influence of interaction between charged particles and dielectric surface over a homogeneous barrier discharge in nitrogen. J. Phys. D. Appl. Phys. 35, 751 (2002).

46. Behnke, Y. B. G. and V. A. M. and J. B. and J. F. On the stability of a homogeneous barrier discharge in nitrogen relative to radial perturbations. $J$. Phys. D. Appl. Phys. 36, 975 (2003).

47. Xu, S.-W., He, F., Wang, Y., Li, L. \& Ouyang, J.-T. Effect of volume and surface charges on discharge structure of glow dielectric barrier discharge. Phys. Plasmas 20, 83515 (2013).

48. Hao, Y. et al. Transitions between patterned discharges and diffuse discharges in atmospheric helium under applied voltages far below the discharge inception voltage. Phys. Plasmas 25, 13516 (2018). 